\title{
Magnetic resonance demonstration of gall bladder ascariasis
}

\section{Gaurav Mittal $\cdot$ Ranjana Gupta $\cdot$ Puneet Mittal}

Published online: 17 December 2011

(C) Indian Society of Gastroenterology 2011

A 40-year-old man presented with right hypochondrial and epigastric pain and tenderness. There was no history of jaundice or vomiting. Serum alkaline phosphatase was mildly elevated (170 IU/L). His ultrasound was suggestive of gall bladder worms or stones. MRI revealed curvilinear hypointense structure in the gall bladder (Fig. 1); this suggested the diagnosis of gall bladder ascariasis. Common hepatic and bile ducts were normal. Stool examination revealed ova of Ascaris lumbricoides. The patient was given analgesics and albendazole (400 $\mathrm{mg}$ single dose). Three days later, he reported relief of symptoms and passage of worm in the stools. Ultrasound done 4 days later showed no worm in the gall bladder.

The adult worm of ascariasis usually resides in the small bowel. It can occasionally migrate into biliary system. Involvement of gall bladder is rare because of the small size of cystic duct [1].

Ultrasound is the usual imaging modality for diagnosing gall bladder ascariasis which demonstrates it as curvilinear echogenic structure [1]. In cases which are inconclusive on sonography, MRI may show curvilinear hypointense structure in the gall bladder lumen, while gall stones typically appear as rounded objects of signal void [2]. MRI also allows simultaneous visualization of common hepatic and common bile ducts. Gall bladder ascariasis is usually treated

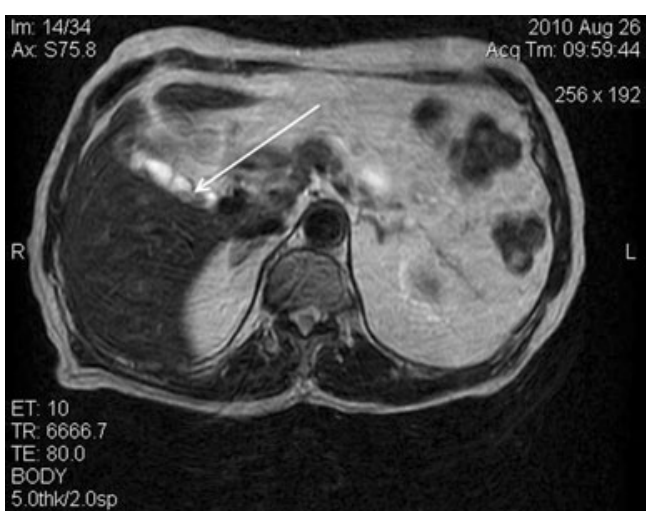

Fig. 1 Axial T2W image shows curvilinear hypointense structure in the gall bladder lumen suggestive of gall bladder ascariasis

by surgery and endoscopy. Occasionally, medical therapy may lead to spontaneous passage of worm, as happened in our case.

\section{References}

1. Khuroo MS, Zargar SA, Yattoo GN, et al. Sonographic findings in gallbladder ascariasis. J Clin Ultrasound. 1992;20:587-91.

2. Arya PK, Kukreti R, Arya M, Gupta SN. Magnetic resonace appearance of gall bladder ascariasis. Indian J Med Sci. 2005;59:208-10.

\section{G. Mittal}

Department of Medicine, Punjab Institute of Medical Sciences,

Jalandhar, India

\section{R. Gupta $\cdot$ P. Mittal $(\bowtie)$}

Department of Radiodiagnosis,

Punjab Institute of Medical Sciences,

Jalandhar, India

e-mail: drpuneetmittal@gmail.com 